\title{
Germanica
}

\section{Lectures marxistes de La Mort de Danton (Büchner) : interprétations de Georg Lukács et Hans Mayer autour de 1935 et leur réception en RDA}

Marxistische Lektüren von Dantons Tod (Büchner): Interpretationen von Georg Lukács und Hans Mayer um 1935 und deren Rezeption in der DDR Marxist literary criticisms on Danton's Death (Büchner): Interpretations by Georg Lukács and Hans Mayer in the mid-1930s and their reception in the GDR

Laetitia Devos

\section{OpenEdition}

Journals

Édition électronique

URL : http://journals.openedition.org/germanica/3363

DOI : 10.4000/germanica.3363

ISSN : 2107-0784

Éditeur

Université de Lille

Édition imprimée

Date de publication : 30 décembre 2016

Pagination : 51-65

ISBN : 9782913857384

ISSN : 0984-2632

\section{Référence électronique}

Laetitia Devos, « Lectures marxistes de La Mort de Danton (Büchner) : interprétations de Georg Lukács et Hans Mayer autour de 1935 et leur réception en RDA », Germanica [En ligne], 59 | 2016, mis en ligne le 30 décembre 2018, consulté le 06 janvier 2021. URL : http://journals.openedition.org/germanica/ 3363 ; DOI : https://doi.org/10.4000/germanica.3363 


\title{
Lectures marxistes de La Mort de Danton (Büchner) : interprétations de Georg Lukács et Hans Mayer autour de 1935 et leur réception en RDA
}

\author{
Laetitia DEVOS \\ Rennes 2, ERIMIT/GRAAL
}

Georg Büchner, qui vécut de 1813 à 1837 et fut redécouvert seulement à la fin du XIXe siècle ${ }^{1}$, fut considéré moins d'un siècle après sa mort comme l'un des précurseurs de la modernité. Son œuvre inspira Gerhart Hauptmann, Frank Wedekind, Ernst Toller, Bertolt Brecht, Peter Weiss, Volker Braun ou encore Heiner Müller. L'anthologie en trois tomes rassemblée par Dietmar Goltschnigg sur « Büchner et la modernité 2 » est la preuve, s'il en fallait, de la fécondité de la référence à Büchner au XX ${ }^{\mathrm{e}}$ siècle.

1. - Léonce et Léna fut créé le 31 mai 1895 à Munich au cours d'une représentation privée en plein air. La pièce ne fut jouée à nouveau qu'à partir de 1912 (Düsseldorf). La Mort de Danton fut donnée une première fois par des étudiants amateurs à ZürichFluntern au début des années 1890, puis créée en janvier 1902 au Belle-Alliance-Theater de Berlin. La première représentation de Woyzeck (orthographié Wozzeck à l'époque) eut lieu en 1913 au Residenztheater de Munich.

2. - Dietmar Goltschnigg (éd.), Georg Büchner und die Moderne. Texte, Analysen, Kommentar, 3 volumes (vol. 1 : 1875-1945, vol. 2 : 1945-1980, vol. 3 : 1980-2000), 
Ce qui le rattache à la modernité tient tout d'abord à la forme de ses œuvres : le «puzzle » qu'a constitué Woyzeck pendant de nombreuses années, avant que les scientifiques n'arrivent à déterminer de façon à peu près certaine l'ordre exact dans lequel l'auteur avait placé les scènes $^{3}$, a alimenté le mythe d'une pièce à géométrie variable qui a séduit des écrivains dramatiques à la recherche de nouvelles formes. La non-conventionalité concerne aussi le drame La Mort de Danton, conçu de manière originale en quatre actes, sans respect pour le schéma classique reposant sur une exposition, des péripéties et un dénouement. À l'inverse, l'hyper-conventionalité de Léonce et Léna dénonce les formes normatives de la comédie. D'autres caractéristiques encore rattachent Büchner à la modernité, au premier rang desquelles l'absence de point de vue surplombant, malgré une présence indéniable de l'auteur qui épouse certains discours pour mieux les démonter et les renvoyer dos à dos. Le questionnement permanent et la remise en question de toutes les certitudes contribuent au brouillage des pistes typique des drames modernes. C'est ainsi que Georg Büchner fut l'un des auteurs dramatiques les plus joués sous la République de Weimar ${ }^{4}$.

Sous le national-socialisme, les intellectuels en exil s'interrogèrent sur un patrimoine culturel allemand propre à lutter contre le nazisme, et, au-delà, à fonder une société socialiste. Des points de vue divergents s'affrontèrent sur la place à accorder à la modernité, et plus précisément à l'expressionnisme dans la culture allemande. Le philosophe Georg Lukács soutenait que l'expressionisme avait, involontairement, favorisé l'ascension du fascisme en alimentant la thèse d'un monde soumis à des forces obscures, irrationnelles et insaisissables. Or, Büchner avait été une source d'inspiration indéniable pour les expressionnistes et sa réception théâtrale était nourrie de cette imprégnation ${ }^{5}$. Était-il dès lors possible pour Lukács de condamner l'expressionnisme tout en gardant Büchner dans le patrimoine culturel socialiste ? Il sera plus particulièrement question ici du drame La Mort de Danton (1835), drame de la Révolution française revisitée par Büchner à une époque de Restauration.

Berlin, Erich Schmidt Verlag, 2001-2004.

3. - Un jalon important est l'édition des facsimilés en 1981 par Gerhard Schmid. En 1990, Thomas Michael Mayer proposa une nouvelle édition et à sa suite en 1992 Henri Poschmann. La version qui fait aujourd'hui foi est celle de l'édition critique de Marbourg établie par Burghard Dedner.

4. - Ulrich Kaufmann, Dichter in «stehender Zeit ». Studien zur Georg-BüchnerRezeption in der DDR, Erlangen, Palm und Enke, 1992, p. 24. Wolfram Viehweg, Georg Büchners «Dantons Tod » auf dem deutschen Theater, München, Laokoon, 1964.

5. - W. Viehweg, Georg Büchners..., op. cit., p. 20 sq. 


\section{Les pierres d'achoppement de La Mort de Danton : le fatalisme de Danton et le discours de Saint-Just}

De nombreux commentateurs, au premier rang desquels Hans Mayer, ont relevé que Büchner avait transformé le Danton historique, qui avait péché par excès de compromis, en un Danton fataliste, fatigué de vivre, renonçant à toute action face au caractère insondable du destin. Ainsi à la scène 5 de l'acte II (scène du cauchemar), Danton déclare :

L'homme sur la croix a eu la partie belle : il faut que le scandale arrive, mais malheur à celui par qui le scandale arrive. Il faut, c'était ce même « il faut ». Qui maudira la main sur laquelle est tombée la malédiction du « il faut »? Qui a dit « il faut », qui 6 ?

La question qui se pose est de savoir si Büchner fait de Danton son porte-parole à ce moment-là. En effet, une lettre qu'il a adressée à sa fiancée recèle la même citation de l'Évangile selon saint Matthieu $(18,7)$, soulignée par nous ici :

Il faut est l'une des paroles de condamnation avec lesquelles l'homme a été baptisé. Le mot selon lequel il faut certes que le scandale arrive, mais malheur à celui par qui il arrive - a de quoi faire frémir. Qu'est-ce qui en nous ment, assassine, vole? Je n'ai pas envie de suivre plus avant cette idée?

Cette proximité entre la plainte de Danton et la lettre de Büchner pose la question de savoir si l'auteur adhère au fatalisme de son personnage et s'il affirme qu'il est impossible d'influer sur le cours de l'Histoire.

Une question corollaire est celle de l'interprétation du discours de Saint-Just (II, 7), qui exprime moins un fatalisme comme celui de Danton qu'un déterminisme historique, comparable à la nécessité naturelle telle qu'elle a été développée par Holbach ${ }^{8}$. Saint-Just déclare :

[...] nous ne sommes pas plus cruels que la nature et que le temps. Calme et inexorable, la nature suit ses lois, et l'être humain qui entre en conflit avec celles-ci est anéanti. Une modification des composants de l'air, un embrasement tellurique, un brusque déséquilibre d'une masse d'eau, et aussitôt une épidémie, une éruption volcanique, une inondation font périr les hommes par milliers. Et quel est le résultat ? Une modifica-

6. - Traduction de Jean-Louis Besson et Jean Jourdheuil, Montreuil, éditions théâtrales, 2005. Toutes les citations de La Mort de Danton seront tirées de cette traduction.

7. - Georg Büchner, Correspondance, traduction de Henri-Alexis Baatsch, préface de Jean-Christophe Bailly, Paris, Union générale d'édition, 1974.

8. - Rapprochement proposé par Bodo Morawe, Faszinosum Saint-Just. Zur programmatischen Bedeutung der Konventsrede in Danton's Tod $(I I, 7)$ von Georg Büchner, Bielefeld, Aisthesis, 2012, p. 11 sq. 
tion insignifiante de la nature physique, somme toute à peine perceptible, et qui se serait produite sans presque laisser de traces s'il n'y avait des cadavres sur son chemin.

Je demande donc : pourquoi la nature morale, dans ses révolutions, devrait-elle avoir plus d'égards que la nature physique ? Pourquoi une idée, tout aussi bien qu'une loi physique, n'aurait-elle pas le droit d'anéantir ce qui s'oppose à elle ? Pourquoi un événement qui transforme l'organisation tout entière de la nature morale, c'est-à-dire de l'humanité, ne s'accomplirait-il pas dans le sang ? L'esprit du monde utilise nos bras dans la sphère spirituelle, tout comme il se sert des volcans et des inondations dans la sphère physique. Qu'importe qu'ils meurent d'une épidémie ou de la Révolution ! [...] La Révolution est comme les filles de Pélias ; elle dépèce l'humanité pour la rajeunir.

Le Saint-Just de Büchner considère que la révolution est aussi nécessaire que le sont les révolutions de la nature. Les origines de ce discours sont multiples et l'édition critique de Marbourg les a recensées : la source assurément la plus proche est celle de Charles Nodier qui retranscrit lui-même un discours tenu par Pierre Vergniaud. Büchner emprunte au discours de Vergniaud des références bibliques (Moïse) et mythologiques (les filles de Pélias) qu'il place dans la bouche de SaintJust $^{9}$. Hormis la toute dernière exhortation à l'action que Saint-Just adresse aux « ennemis secrets de la tyrannie qui portent le poignard de Brutus sous leur habit », que l'on retrouve dans le discours historique de ce même personnage retranscrit par la revue Unsere Zeit ${ }^{10}$, Büchner invente entièrement le discours de Saint-Just, ou plus exactement, il emprunte et met bout à bout des motifs de discours trouvés dans les revues et ouvrages qu'il a consultés sur la Révolution française et tenus davantage par Robespierre (notamment l'analogie entre la nature physique et la nature « morale $\left.{ }^{11} »\right)$ que par Saint-Just.

C'est dans ce jeu intertextuel que naît la polysémie du discours de Saint-Just. En effet, pour qui ne connaît pas les sources de Büchner, la chute du discours, la comparaison avec les filles de Pélias, peut apparaître comme un commentaire ironique de l'auteur sur son personnage, venant invalider ce qui précède : involontairement, Saint-Just annoncerait l'échec de la Terreur puisque l'on sait que les filles de Pélias ont échoué à rajeunir leur père en le dépeçant. Mais ce n'est pas en ce sens que Vergniaud, personnage auquel Büchner emprunte la comparaison, a proposé la référence aux Péliades. Nodier retranscrit la phrase précise de Vergniaud : «Les Péliades, qui égorgèrent leur vieux père pour le

9. - Burghard Dedner, Thomas Michael Mayer, Eva-Maria Vering, Danton's Tod. Historische Quellen, édition critique de Marbourg, Darmstadt, Wissenschaftliche Buchgesellschaft, 2000, vol. 3.3, p. 350.

10. - Ibid., p. 215.

11. - Ibid., p. 200, p. 237-238. 
rajeunir étaient d'habiles républicaines. Elles savaient le secret des révolutions ${ }^{12}$ ». Vergniaud faisait l'apologie de la tabula rasa, car elle était à ses yeux une étape sine qua non de la révolution. Une autre source de cette comparaison pourrait venir de Hobbes et de Diderot qui ont tous les deux également utilisé la référence aux Péliades pour étayer des thèses pourtant opposées : le premier pour condamner les excès révolutionnaires, et le deuxième pour justifier l'éradication radicale des sociétés corrompues ${ }^{13}$.

Pour qui connaît ces différentes sources, il apparaît clairement que Büchner condense dans le discours de Saint-Just, par la référence aux Péliades, une controverse sur les limites à fixer (ou non) au mouvement révolutionnaire. La tirade de Saint-Just peut servir à alimenter la thèse des partisans d'une révolution modérée comme celle des partisans d'une révolution radicale. Büchner brouille les pistes et c'est ce qui fait la résistance de sa pièce à toute interprétation dogmatique. Nous nous proposons d'étudier l'interprétation que Lukács et Mayer donnèrent à cette pluralité de sens, qui fait toute la modernité de Büchner, pendant leur exil sous le national-socialisme.

\section{Lukács : «Le Büchner fascisé et le vrai Büchner » (1937)}

Lukács publia en 1937 dans la revue du front antifasciste Das Wort un article intitulé «Der faschisierte und der wirkliche Georg Büchner ${ }^{14} »$. Cet article visait, comme l'indiquait son titre, une réhabilitation du « vrai » Büchner, après sa déformation sous le « $\mathrm{III}^{\mathrm{e}}$ Reich ». Lukács commençait donc par rappeler que sous le nazisme, le drame $L a$ Mort de Danton était considéré comme l'expression de la désillusion d'un révolutionnaire. Les nationaux-socialistes entretenaient la thèse du révolutionnaire déçu, nihiliste, qui regardait dans un vide auquel seul le national-socialisme pouvait donner une alternative. Lukács assimilait, de ce point de vue, un peu rapidement les philologues Arthur Pfeiffer et Karl Viëtor. Hans Mayer réhabilitera le deuxième dans sa deuxième édition (1960) de Georg Büchner et son temps.

12. - Ibid., p. 350.

13. - Références relevées et explicitées par Bodo Morawe, Faszinosum, op. cit., p. 61-85. Morawe affirme cependant que selon la transcription de Nodier, le SaintJust historique aurait lui aussi comparé la Révolution aux filles de Pélias. D'après nos recherches en revanche, les extraits de l'ouvrage de Nodier publiés dans l'édition critique de Marbourg attribuent le discours à Vergniaud.

14. - Georg Lukács, « Der faschisierte und der wirkliche Georg Büchner. Zu seinem hundertsten Todestag », Das Wort, ${ }^{\circ} 2$, 1937, p. 7-26. Paru aussi dans id., Deutsche Realisten des 19. Jahrhunderts, Berlin, Aufbau, 1951, p. 66-88 et dans Wolfgang Martens (dir.), Georg Büchner, Darmstadt, Wissenschaftliche Buchgesellschaft, 1965, p. 197-224. C'est la pagination de l'article original que nous citerons. 
Un parti pris méthodologique de Lukács est de refuser de distinguer entre le personnage " public » qui s'engage pour la révolution et un Büchner qui, dans un second temps de sa vie, aurait perdu ses convictions révolutionnaires et exprimerait son désarroi dans ses œuvres dramatiques. Büchner est une seule et même personne. Il ne s'agit pas de nier certaines contradictions, mais de les éclairer par le contexte de l'époque.

Lukács s'inscrit en opposition avec ce qu'il dénonce comme le " Büchner fascisé », qui serait un Büchner nihiliste, et pour cela il commence par la biographie de l'auteur : il rappelle ses actions révolutionnaires, son engagement dans la Société des droits de l'homme, la périlleuse publication et distribution de son libelle Le Messager hessois. Il conclut de cette biographie que Büchner appartient à la lignée révolutionnaire qui va de Babeuf à Blanqui, mais qu'il ne pouvait encore percevoir la classe que formait le prolétariat, qui s'appelait encore « les pauvres ». Pour Lukács, dans La Mort de Danton, le peuple est central et anticipe l'avenir : il montre que la révolution n'est pas finie, contrairement à ce que prétend Danton. Cependant, il ne réussit pas à agir véritablement, en raison de son absence de conscience de classe.

Ainsi, selon Lukács, Büchner vit une époque charnière de l'Histoire, la crise de transition des visions révolutionnaires : d'un côté, la Révolution française déçoit car elle n'a pas amélioré la situation du peuple, mais de l'autre, certains sont prisonniers de « l'illusion» (le mot est de Lukács ${ }^{15}$ ) qu'en menant la terreur jacobine à son terme, on finira par libérer le peuple de sa misère matérielle. Lukács étudie l'antagonisme des deux personnages principaux et considère que sur le plan strictement politique Büchner donne l'ascendant à Robespierre, auquel Danton n'a rien à opposer. Selon Lukács, Danton fait l'erreur de considérer que la révolution est achevée dès lors que la société s'est libérée du joug de la féodalité, et ne perçoit pas une deuxième étape, celle de l'affranchissement du capitalisme. Son ennui est celui de la bourgeoisie de l'après-Révolution française, rassasiée et inactive. La révolution devient étrangère à Danton, elle lui échappe, d'où son fatalisme.

Sur la question du fatalisme, Lukács affirme que Büchner se distingue en ceci de Danton qu'il ne voit pas dans les événements une fatalité mais une "nécessité historique ». Citant la célèbre lettre dite «sur le fatalisme », il en souligne les différences avec la complainte de Danton. Dans sa lettre, Büchner ajoute que si on ne peut maîtriser la nécessité historique, la « connaître reste le but ultime ». Lukács en conclut :

C'est pourquoi ce « il faut » de Büchner n'est pas désespéré, n'est pas pessimiste, au contraire de celui de Danton. Dans le 
drame, Büchner répond de façon structurée aux doutes de Danton dans le grand discours de Saint-Just devant la Convention, discours dans lequel la dure et inhumaine nécessité de l'Histoire [...] est approuvée et glorifiée avec passion et pathos ${ }^{16}$.

Selon Lukács, le pessimisme de Danton est révélateur de « l'incapacité de l'ancien matérialisme à comprendre l'Histoire » et Büchner adhérerait à la conception de Saint-Just qui présente la violence révolutionnaire comme une nécessité aussi inéluctable qu'une révolution de la nature.

Jusque-là, la thèse de Lukács, réputé pour ses " grilles de lecture » idéologiques, ne nous surprend pas. Toutefois, son article ne se limite pas à l'aspect politique : ce qui est important et qui sera passé sous silence en RDA par les interprètes dogmatiques qui prétendaient pourtant le citer, c'est que l'idéologue hongrois ne nie pas l'ambiguïté de la position de Büchner. Il reconnaît que « Büchner partage les visions philosophiques épicuriennes du monde de Danton » et qu'il éprouve une «sympathie dramatico-lyrique » pour ce personnage. Il ne fait pas de lui un contre-révolutionnaire :

Le Danton de Büchner n'est pas un bourgeois réactionnaire. Il se moque avec cynisme de la théorie qu'a Robespierre de la morale - mais il n'éprouve aucune sympathie pour ses partisans (sauf pour Camille Desmoulins). Pour quelle cause peut-il se battre ? Avec qui se battre ? Son partisan Lacroix se désigne lui-même comme une canaille ; le général Dillon veut libérer Danton avec l'aide des partisans suivants: « Je trouverai assez de personnes, des vieux soldats, des girondins, des anciens nobles ». Que le Danton de Büchner refuse justement un combat au côté de tels alliés montre que l'idée révolutionnaire est restée intacte en lui ${ }^{17}$.

Finalement, Lukács en arrive à la conclusion que Büchner distribue ses propres réflexions entre Danton d'un côté et Saint-Just et Robespierre de l'autre :

Seuls les deux ensemble, dans leur interaction tragique, incarnent les idées de Büchner, ni Danton ni Saint-Just ne sont en soi un porteparole de l'auteur. Toutefois, la conception de Saint-Just est celle qui se rapproche le plus de la manière dont Büchner envisage de résoudre la « question de la faim ». Il est vrai que tant Robespierre que SaintJust ont des caractéristiques dont nous trouvons des traces lyriques dans le discours de Büchner sur Caton. Mais Robespierre et Saint-Just sont aussi peu identifiables à Büchner que Danton. Et c'est justement 
parce que Büchner, dans cette grande crise intellectuelle, reste imperturbablement attaché à la philosophie matérialiste et ne perd jamais la conviction que cette philosophie permettra de résoudre les grands problèmes de la vie que Danton est plus proche de son sentiment que ne l'est Saint-Just, pourtant apparenté politiquement ${ }^{18}$.

Selon Lukács, Büchner voit dans le matérialisme sensualiste de Danton un contrepoint absolument nécessaire à l'idéalisme moralisateur de Robespierre, même s'il aboutit à une aporie qui prouve que ce matérialisme sensualiste est dépassé. L'attirance de Büchner pour le matérialisme de Danton n'est pas tue, Lukács n'a de cesse de la souligner, car c'est ce déchirement de l'auteur entre plusieurs tendances en apparence inconciliables qui l'intéresse. Et en cela, Büchner aurait affaire aux questionnements et déchirements qui seront ceux de Marx et Engels après lui : «Le jeune Marx et Engels sont encore obligés, alors qu'ils se situent déjà sur le terrain du matérialisme dialectique, de combattre la conception ascétique de la révolution ${ }^{19}{ }^{»}$.

Lukács fait de Büchner un précurseur immédiat de Marx et Engels, sans taire les contradictions inhérentes à son œuvre et qu'il explique par le contexte :

Il va de soi que Büchner ne parvient pas à trouver une solution, car ce qu'il cherche politiquement, la concrétisation des « pauvres » en «prolétariat», n'est pas possible dans la réalité allemande de son temps ${ }^{20}$.

Dans les deux premières décennies de la RDA, les exégètes dans la droite ligne du Parti (par exemple Alfred Kurella ou Alexander Dymschitz) retiendront de Lukács exclusivement l'idée que Büchner était un précurseur de Marx et que les questions qu'il laissait irrésolues étaient liées au fait qu'il ne pouvait encore percevoir la lutte des classes. Lorsqu'ils citent Lukács, les idéologues dogmatiques se limitent à cette paraphrase et déforment le propos pour présenter Danton en contrerévolutionnaire. Le nom de Lukács servit à accréditer la théorie selon laquelle Büchner donnait intégralement l'ascendant à Saint-Just, ce que nuance Lukács justement.

L'approche socio-historique, qui consiste à analyser l'œuvre dans son contexte, est également celle de Hans Mayer. Toutefois, les idéologues dogmatiques peinèrent davantage encore à l'instrumentaliser à des fins politiques.

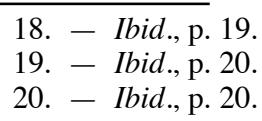

19. - Ibid., p. 20.

20. - Ibid., p. 20. 


\section{Hans Mayer : «atmosphère thermidorienne »(1935-39)}

Hans Mayer commença à écrire son ouvrage Büchner et son temps en exil, en 1935, deux ans avant la publication de l'article de Lukács. Il l'acheva en 1939 et ne le retoucha, pas même s'il ne put le publier qu'en 1946 à l'Ouest (aux éditions Limes) et en 1947 à l'Est (aux éditions Volk und Wissen). Le sous-titre du chapitre consacré à La Mort de Danton est « atmosphère thermidorienne ${ }^{21}$ ».

Mayer part d'un postulat identique à celui de Lukács : il se refuse à distinguer le Büchner-écrivain du Büchner-révolutionnaire et il consacre les premières pages du chapitre à resituer le contexte dans lequel Büchner a écrit son drame. Mais il se méfie également d'une lecture purement idéologique et il annonce d'emblée qu'il se refuse à voir dans l'œuvre dramatique de l'écrivain des « pièces à thèse » :

Ce qui est déterminant, c'est la question de l'unité. Partir de l'aspect politique pour la reconstituer, ne veut par exemple pas dire assujettir l'écriture et la pensée à une loi étrangère ; il ne s'agit pas d'interpréter les textes comme des pièces " à thèse ", ce qu'ils ne sont vraiment pas au sens le plus courant du mot ${ }^{22}$.

Mayer veut expliquer l'œuvre de Büchner par l'antagonisme de son époque : comme Lukács, il voit dans le Vormärz une époque-charnière marquée par des changements sociopolitiques qui ne permettaient pas encore d'entrevoir la nouvelle classe, le prolétariat industriel. Il insiste sur le caractère dialectique de l'œuvre de Büchner, à la fois aboutie pour son époque et ouverte. Büchner était parvenu aux limites de ce qu'il était possible de concevoir à l'époque.

Après avoir resitué La Mort de Danton dans l'atmosphère de précipitation et d'angoisse dans laquelle Büchner écrivit ce drame, il l'interprète comme une mise en question de la possibilité même de révolution :

Danton retravaille l'ensemble des expériences pratiques et politiques de Büchner, pas seulement la peur et l'inquiétude des dernières semaines avant sa fuite. [...] C'est l'expression de toute une révolution qui a échoué, pas seulement l'échec d'un seul révolutionnaire. [...] Il n'embrasse pas moins que toute la question des possibilités et limites de la révolution politique et sociale ${ }^{23}$.

21. - Hans Mayer, " Thermidorstimmung ", Georg Büchner und seine Zeit, Frankfurt a.M., Suhrkamp, 1972, p. 200-223.

22. - Ibid., p. 22. Le premier chapitre de l'ouvrage, « fragment ou achèvement », pose les jalons méthodologiques de l'étude.

23. - Ibid., p. 204. 
Sur la question du fatalisme de Büchner, l'interprétation de Hans Mayer diffère de celle de Lukács : il est convaincu de l'adhésion parfaite de l'auteur et de Danton dans la scène du cauchemar (II, 5) : Danton serait le porte-parole incontestable de Büchner à ce moment précis.

C'est ainsi que Hans Mayer, pour prouver l'identification au moins partielle de l'auteur et de Danton, relève toutes les inventions de Büchner dans le personnage : son inaction, son scepticisme quant au sens même de la révolution sont autant de traits que Büchner donne a posteriori au révolutionnaire, qu'il reconstruit de la perspective de Thermidor.

D'un autre côté, Mayer met en garde contre une interprétation « psychologique superficielle » qui consisterait à assimiler intégralement Danton à Büchner : il rappelle que certains discours tenus par Danton, notamment son discours de défense au tribunal, sont repris mot pour mot des sources historiques et qu'il ne faut donc pas y voir le discours que Büchner comptait tenir s'il était arrêté, selon une hypothèse hasardeuse.

Pour ce qui est du personnage de Saint-Just, Mayer relève un gouffre cette fois indubitable qui sépare le personnage historique du personnage inventé par Büchner. Le discours que Büchner lui fait tenir à la scène 7 de l'acte II est non seulement inventé, mais à l'opposé de ce qu'aurait dit le personnage historique. En effet, selon Mayer, le Saint-Just historique, dans son discours à la Convention, avait condamné la vision fataliste de l'Histoire. Dans son réquisitoire contre Danton, il n'avait non seulement pas repris à son compte la comparaison de la Révolution avec Saturne qui dévorait ses propres enfants ${ }^{24}$, mais il avait au contraire dénoncé cette comparaison, considérant que le fatalisme était invoqué par ceux qui tremblaient pour se disculper. Le Saint-Just historique avait récusé la vision fataliste en affirmant que « la République ne dévorera[it] pas ses enfants, mais ses ennemis ». Selon Mayer, condamner Danton était un acte de liberté et non une soumission au déterminisme de l'Histoire. Mayer considère que Büchner inverse sciemment le sens du discours de Saint-Just pour véhiculer sa propre conception pessimiste de l'Histoire :

La réinterprétation des faits historiques, inspirée de l'atmosphère thermidorienne, n'est peut-être à aucun autre moment plus claire qu'ici dans la caractérisation de Saint-Just. Il est très significatif que Büchner prenne ses libertés vis-à-vis des sources historiques dans le discours de Saint-Just devant la Convention, et qu'il trouve et construise lui-même le discours, certes à l'aide de mots typiques de Saint-Just, mais dans un esprit totalement opposé au discours d'accusation historique que Saint-

24. - Mayer attribue cette comparaison à Saint-Just. Dans le drame de Büchner, c'est Danton qui prononce cette phrase à la scène $\mathrm{I}, 5$. 
Just tint contre Danton ! À ce moment-là, la position fondamentale du drame est parfaitement visible 25 .

Mayer l'exprime à nouveau, plus simplement : «Büchner voit Thermidor et son inévitabilité26 ». Par la voix de Saint-Just, Büchner exprimerait son fatalisme, comme il l'avait fait dans la scène du cauchemar de Danton. Mayer soutient que La Mort de Danton véhicule une vision pessimiste de l'Histoire de la Révolution française, car fondée sur le point de vue d'un jeune Allemand en 1835, en pleine Restauration. Ce serait une déclaration d'impuissance de l'individu, soumis à des « facteurs qui résident hors de lui ». En cela, «La Mort de Danton annonce Woyzeck ${ }^{27} »$.

Là où Lukács interprétait la tabula rasa proposée par Saint-Just comme une étape historique nécessaire à la révolution, Mayer y lit un message pessimiste de l'auteur qui ne voit pas d'issue : la Révolution a échoué, comme les filles de Pélias ont échoué à rajeunir leur père. Hans Mayer ne fait donc pas de Saint-Just une figure positive, porteuse d'espoir. Le fait que Büchner laisse des questions ouvertes fait l'intérêt de la pièce et est représentatif du questionnement de toute une génération : " Une génération se demande pourquoi elle vit et où elle va - et la question reste ouverte. On vit dans une époque de transition sociale et on le sait 28 ».

En RDA, des interprètes dogmatiques usèrent à mauvais escient du constat établi par Mayer pour prétendre corriger le discours sanguinaire de Saint-Just dont l'extrémisme pouvait être lu à double sens.

\section{Réception des études de Lukács et Mayer en RDA : du « lissage » à l'explosion de la modernité}

Sous le régime est-allemand à ses débuts, Lukács et Mayer furent des interprètes aux noms incontournables. Toutefois, leurs analyses étaient plus souvent paraphrasées avec inexactitude que citées in extenso. Elles n'étaient pas assez dogmatiques et s'accordaient mal avec la prise de parti en littérature prônée par le SED. Ainsi, en janvier 1956, Lukács, invité au $4^{\mathrm{e}}$ congrès des écrivains de la RDA, fit-il porter son intervention sur " le problème de la perspective ", exposé au cours duquel il stigmatisa la littérature contemporaine est-allemande, le penchant de cette dernière pour le naturalisme - qu'il distinguait du réalisme - et pour la prise de parti, qui, à ses yeux, nuisait à la représentation de la

\footnotetext{
25. - Ibid., p. 220.

26. - Ibid., p. 221.

27. - Ibid., p. 223.

28. - Ibid., p. 27.
} 
«totalité29 ». De même, Hans Mayer mit le régime dans l'embarras en dénonçant la pauvreté de la création littéraire contemporaine et en en rejetant la faute sur la condamnation de la modernité littéraire par des idéologues comme Alfred Kurella ${ }^{30}$.

Les événements hongrois de 1956 conduisirent à évincer Lukács du paysage politique est-allemand. Le 21 juillet 1957, Kurt Barthel ${ }^{31}$ demanda dans le Neues Deutschland à ce que tous ceux qui avaient partagé les points de vue (même littéraires) du « contre-révolutionnaire » Lukács se rétractent publiquement. Ses ouvrages furent retirés de la circulation. C'était le début de deux longues décennies d'opprobre.

À l'été 1963, ce fut Hans Mayer qui quitta la RDA, las qu'il était des difficultés qu'il rencontrait, dont la dernière était l'interdiction de se rendre à Prague pour un colloque sur Kafka. La thèse du «drame du Thermidor », soutenue par Mayer, fut revue et corrigée dans une série d'articles publiés au sujet du drame dans la revue Theater der Zeit en 1959 : le philologue Helmut Rabe, à la recherche de drames oubliés dans le but d'élargir l'héritage culturel est-allemand, attira l'attention sur La Mort de Danton ${ }^{32}$. Helmut Rabe ne se contentait pas d'apporter des rectifications à l'interprétation de Lukács, qui, selon lui, péchait par un manque de précision quant au concept de «matérialisme », mais il critiquait aussi avec virulence l'interprétation de Hans Mayer qu'il n'hésitait pas à assimiler à la «thèse fasciste » de Karl Viëtor ${ }^{33}$. Rabe considérait que ce n'était pas Danton mais sa mort qui primait dans l'œuvre, et que le personnage le plus proche de Büchner était Saint-Just. Dans la même revue ${ }^{34}$, un article signé Renate Zuchardt affirmait qu'il semblait possible de modifier légèrement le texte afin de donner l'ascendant à

29. - Caroline Gallée, Georg Lukács : seine Stellung und Bedeutung im literarischen Leben der SBZ/DDR 1945-85, Tübingen, Stauffenberg, 1996, p. 95. Voir Neues Deutschland, 13/01/1956 et 16/01/1956.

30. - Allocution radiophonique du 28 novembre 1956 retranscrite dans « Zur Gegenwartslage unserer Literatur », Sonntag, 49/1956 (nov.), p. 4.

31. - Kurt Barthel (qui se surnommait «Kuba ») fut, de 1950 à 1958, député de la Volkskammer, de 1952 à 1954 Premier Secrétaire de l'Union des écrivains, puis membre de son bureau directeur. À partir de 1954, il fut aussi membre du Comité central du SED et de la Commission culturelle au sein du bureau politique du Parti. À partir de 1957, il fut « dramaturge » (au sens allemand du terme) au théâtre de Rostock.

32. - Helmut Rabe, « Georg Büchners Drama Dantons Tod», Studien 12, Beilage zu Theater der Zeit, 14/7/1959, p. 15-23.

33. - « Hans Mayer renoue plutôt avec les tentatives d'interprétation de Karl Viëtor dans la Tragédie du pessimisme hérö̈que. Lukács, au moins, avait essayé dans son essai de contredire la légende de "l'échec du révolutionnaire Büchner" propagée par la bourgeoisie fasciste. Hans Mayer trouve justement dans cette légende le point de départ de son interprétation. »Ibid., p. 16.

34. - Renate Zuchardt, " "Wagt !" - lehrt uns Danton. Vorschläge zur szenischen Realisierung des Büchner-Dramas », Theater der Zeit, 14/7/1959, p. 13-19. 
Robespierre sur Danton. Des corrections, des suppressions et des réorganisations étaient proposées, visant à éliminer ce qui ternissait l'image de Robespierre, notamment la jalousie qu'il semblait éprouver envers Danton. Il fallait également changer l'issue de la pièce, afin que les spectateurs ne repartent pas avec un sentiment de pitié envers les exécutés, mais en ayant en tête les affirmations de Robespierre et de Saint-Just, auxquels il convenait de donner plus de volume de parole.

Ces articles donnèrent l'impulsion à Kurt Barthel qui réalisa une mise en scène de La Mort de Danton en 1962 à Rostock. Il élimina toute la métaphorique sexuelle qu'il trouvait dégradante pour la politique, enrichit le peuple de sans-culottes qui entonnaient des chants révolutionnaires, rétablit le discours historique tenu par Saint-Just et fit de Robespierre comme de Saint-Just des héros, et inversement, de Danton un traitre et renégat.

Alors que la critique se montra unanimement élogieuse ${ }^{35}$ à l'égard de la version de Kuba qui déformait tant la lettre que l'esprit büchnériens dans un sens dogmatique, à la même époque, la réédition (1960) de l'ouvrage de Hans Mayer sur Büchner se heurta à une vive critique. Jürgen Geerdts par exemple, saisit l'occasion d'un éloge qu'il adressa en 1963 dans les Weimarer Beiträge à l'interprétation de la pièce par Kuba, pour souligner que la version de Rostock venait non seulement invalider les interprétations nihilistes, existentialistes et antirévolutionnaires telles qu'elles avaient cours en RFA, mais aussi rectifier la thèse de Hans Mayer lui-même ${ }^{36}$. Selon Geerdts, Hans Mayer s'était mépris sur le rôle du peuple dans La Mort de Danton. Geerdts insistait sur la lignée directe qui menait de Büchner à Marx, alors que Hans Mayer s'était justement efforcé de donner une vision plus dialectique de cette filiation ${ }^{37}$.

Malgré le « lissage » de l'interprétation de Büchner proposée dans les années 1950 et 1960 en RDA, les interprétations de Lukács et de Mayer continuaient à être connues. Pour les intellectuels et les artistes en butte au régime est-allemand, Büchner resta discrètement une figure tutélaire et les exégèses que Lukács et Mayer avaient livrées de son œuvre sans en aplanir les contradictions permirent de renouer, dès qu'on le put, avec ses œuvres dans leur authenticité. Des écrivains comme Volker

35. - D. Goltschnigg (éd.), Georg Büchner und die Moderne..., op. cit., vol. 2, p. 33.

36. - Hans Jürgen Geerdts, «Zum 150. Geburtstag des Dichters am 17. Oktober $1963 »$, Weimarer Beiträge, 1963/9, p. 642-649. Reproduit dans D. Goltschnigg, Georg Büchner und die Moderne, op. cit., vol. 2, p. 327-332.

37. - Dans le même esprit, l'officier russe Alexander Dymschitz publia en 1962 un article qui déplorait que Hans Mayer ne souligne pas le « caractère prémarxiste » de Büchner. Alexander Dymschitz, « Die ästhetischen Anschauungen Georg Büchners », Weimarer Beiträge, 1961, 8/1, p. 108-123. 
Braun, Christa Wolf ou Heiner Müller ${ }^{38}$ (les deux premiers avaient été étudiants de Hans Mayer à Leipzig) firent des parallèles explicites entre la situation de l'écrivain du Vormärz et la leur.

La réception artistique de Büchner permit de retrouver le chemin de la modernité : ainsi, quatre opéras créés en RDA d'après la vie et l'œuvre de Büchner entre 1979 et 1989 (dont trois furent composés quasiment simultanément entre 1978 et 1981) rompaient avec l'héritage dit « classique et humaniste » qui avait été si longtemps à l'ordre du jour sur la scène lyrique, et ouvraient la page de l'avant-garde en RDA, à l'image du compositeur Alban Berg en 1925 avec son Wozzeck ${ }^{39}$.

\section{Conclusion}

Lukács et Mayer livrèrent des avis nuancés sur l'œuvre de Büchner. Ils la louèrent sans en taire les contradictions. Ce sont même ces dernières qui en faisaient l'intérêt à leurs yeux. Tous deux s'accordent à souligner que Danton est en grande partie porte-parole de l'auteur. Lukács considère que la réponse politique au fatalisme de Danton est livrée par le discours de Saint-Just qui compare avec optimisme le déterminisme historique à celui de la nature et croit donc en la nécessaire poursuite de la Terreur. Hans Mayer, quant à lui, lit la tirade de Saint-Just comme un discours bien plus pessimiste de l'auteur : Saint-Just annoncerait, sans le vouloir, sa propre chute et donc Thermidor. Il proclame non la liberté individuelle, mais une force aveugle de l'Histoire, inéluctable. C'est là que transperce le point de vue rétrospectif de Büchner qui projette non seulement sa connaissance de la fin de la Révolution française, mais aussi ses propres échecs en matière de révolution. L'auteur serait face à un abîme et ne le cacherait pas.

Hans Mayer et Lukács expliquaient tous les deux les contradictions de Büchner par l'époque de transition qu'il vivait et qui l'empêchait de distinguer le potentiel de la nouvelle classe sociale qu'allait former le prolétariat. Cette dernière explication fut reprise et amplifiée en RDA et les nuances apportées par les deux philologues évacuées.

On fit dire à Lukács, et dans une moindre mesure à Mayer, ce qu'ils n'avaient pas dit : Kurt Barthel, par exemple, prétendit s'appuyer sur les analyses les plus récentes pour corriger Büchner - dont il affirmait qu'il s'était fourvoyé en réécrivant le discours de Saint-Just - et rétablir le discours historique du personnage. On invoqua régulièrement Lukács

38. - Volker Braun, « Büchners Briefe », Connaissance de la RDA, 1978/7, p. 7-17 (publié en RDA en 1988 seulement). Discours respectifs du prix Büchner de Christa Wolf en 1980 et Heiner Müller en 1985.

39. - Nous renvoyons à notre propre étude : Laetitia Devos, L'opéra en RDA. Sous le signe de Büchner, Rennes, PUR, 2012. 
pour considérer que Saint-Just et Robespierre étaient les porte-parole incontestables de Büchner, ce que Lukács était loin d'avoir affirmé.

Somme toute, on peut dire que les analyses elles-mêmes de Lukács et de Mayer étaient d'une grande modernité pour leur époque, car malgré le contexte de la lutte antifasciste du milieu des années 1930 qui nécessitait de resserrer les rangs, aucun d'eux ne tut les contradictions de $L a$ Mort de Danton. Ils ne plaquèrent pas sur l'œuvre une idéologie toute faite - comme le firent ultérieurement les interprètes « assermentés » de la RDA - mais partirent du « matériau » (l'œuvre) qu'ils mirent en perspective à la lumière du contexte de 1835 et de la biographie de l'auteur. C'est justement ce postulat de départ - l'identité, en dépit de déchirements et contradictions, de l'homme et de l'écrivain - qui fera écho aux questionnements des intellectuels de RDA à la fin des années 1970 et 1980, eux-mêmes à la recherche de formes nouvelles pour des contenus nouveaux. 
